\title{
A Comparative Evaluation Between Intravenous Ketorolac Versus Intramuscular Methadone for Analgesia after Laminectomy
}

\section{Hasan Zabetian, Mohammad Hasan Damshens, Masoud Ghanei, Ahmad Rastgarian*}

Department of Anesthesiology, Critical Care and Pain Management Research Center, Jahrom University of Medical Sciences, Jahrom, Iran.

Study Area: Jahrom, Iran

Coordinates: $28^{\circ} 30^{\prime}{ }^{\prime \prime \prime} \mathrm{N}$; $53^{\circ} 33^{\prime} 38^{\prime \prime} \mathrm{E}$

Key words: Headache, Palpitations, Dry mouth, Nausea, Drowsiness

The permission was obtained from the ethics committee of Jahrom University of Medical Sciences (IR.JUMS.REC.1394.002)

\section{Abstract}

Pain is an unpleasant sensory and emotional experience causes due to tissue damage, which is now used as the fifth vital signs. However, nowadays pain management options are not few, but still, physicians are incapable towards effective control of acute pain, especially postoperative pain. Opioid analgesics are often used for this purpose, which is associated with high risk of side effects. Thus, we are all in search of an alternate method to control pain to reduce the risk of side effects associated with the use of opioids.The present study was conducted on 60 patients randomly divided into two groups (A \& B), who underwent laminectomy in Jahrom Peymanieh Hospital. Group A ( $\mathrm{n}=$ 30) patients received single dose of $30 \mathrm{mg}$ intravenous ketorolac at first and followed by $15 \mathrm{mg}$ intravenous ketorolac at every 6 hours gap for 24 hours. Group B $(n=30)$ patients received $5 \mathrm{mg}$ intramuscular (IM) ketorolac at first and followed by the same dose at every 6 hours for 24 hours. Pain Questionnaire Visual Analog Scale (VAS) and vital sign were evaluated before and after administration of analgesic every 6 hours for 24 hours. Data were collected using questionnaires and analyzed in SPSS18. The results showed that intravenous Ketorolac compared to IM Methadone is more effective in the management of acute postoperative pain and has less complications and less physical dependency. However, unlike methadone, ketorolac did not decrease postoperative morphine usage.

Ketorolac is an NSAID drug that inhibits cyclooxygenase and lipoxygenase (Hyers et al., 1992), releases androgenic opioids (Domer, 1990) and prevents the synthesis of prostaglandins and leukotrienes. Thus, ketorolac seems to be stronger and more effective than other NSAIDs. The effects of ketorolac have extensively been examined in many studies e.g. pains after abdominal, orthopedic, or gynecologic surgeries. Furthermore, ketorolac can also relieve moderate-to-severe pain (Gillis et al., 1997). Methadone is also a synthetic drug with properties similar to that of morphine, which can be administered orally or intravenously. Methadone works for a longer duration as compared to morphine. However, in the case of accurate titration, its sedative effect is lower than morphine (Garrido et al., 1999). Methadone could not be the selected as a drug for a patient having an acute illness and the courses of hospitalization are changing rapidly. improves the quality of life (Halperin et al., 1989). 
Methadone is a good choice for patients who have a long recovery period and for patients whose separation from the ventilator expected to take long (Gillis et al., 1997). Usually, when patients' clinical conditions are stabilized, the change from fentanyl infusion or morphine to IM methadone can help to simplify the care programs and reduce infusion dependence (Gillis et al., 1997). A large part of the spinal surgery is performed with general anesthesia and there is a lack of proper studies on the level of more analgesia of ketorolac compared to other drug groups. Moreover, there have been no studies on the analgesic effect of ketorolac compared to opioids in spinal surgery and no comparative studies on ketorolac analgesia with methadone in spinal surgeries is available so far. The present study was conducted to compare the analgesic effect of methadone with ketorolac in postoperative to the spinal surgery in the surgical ward of Jahrom Peymanieh Hospital.

\section{Methodology:}

We did a clinical trial based study, where 60 patients where considered enrolled for spinal surgery. At first, gender and age were standardized. Prior to the conducting the study. The patients were asked for medical history, especially liver disease, allergy to NSAID drugs, and the use of sedative and anticonvulsants drugs which were recorded. The patients were randomly divided into two groups according to the random numbers table; group 'A' with 30 subjects and group 'B' 30 subjects. Group 'A' patients received a single dose of $30 \mathrm{mg}$ intravenous ketorolac at first and $15 \mathrm{mg}$ intravenous ketorolac at every 6 hours gap for 24 hours. Group 'B' patients received $5 \mathrm{mg}$ IM ketorolac at first then the same was repeated at every 6 hours gap for 24 hours. In order to observe the ethics in research and considering the painful processes in patients with severe unrest and pain and if patient VAS was more than 4 , then 2 $\mathrm{mg}$ of IV morphine was injected if necessary to make VAS less than 4. VAS pain questionnaire recorded the severity of pain and vital signs of the patient at every 6 hours gap for 24 hours continuously. The patients' pain severity was measured according to VAS. VAS is def ined to the patient that according to this criterion, the least pain experienced by the patient in life is zero and the greatest pain 10. The inclusion criteria were age 18 to 58 years, insensitivity to narcotic anesthetics and NSAIDs, no history of hepatic disease, no coagulation diseases, no drug addiction, not being pregnancy and breastfeeding, no history of renal diseases, non-use of analgesics and opioid during the 24 hours prior to surgery, and Class 1 and 2 ASA patients undergoing spinal surgery. The exclusion criteria were patients with hepatic, renal, and cardiovascular diseases and the patients in their pregnancy and breastfeeding, opioid addiction, patients with opioid and NSAID sensitivity, and the patients who had used sedative and opioids 24 hours before surgery. Data were collected using a questionnaire and analyzed in SPSS-18. The percentage was used to describe the quantitative variables converted to their respective mean and standard deviation to describe quantitative variables. In examining the quantitative variables, the normal distribution of the variables was examined using Kolmogorov-Smirnov test and $p$ value $\geq 0.05$ was considered as the normal distribution. On the values of the distribution of the variables, parametric and non-parametric tests were employed to compare the means of each group. Chi-square test was used to examine the qualitative variables. In all statistical analyses, $\mathrm{p}$ value $>0.05$ was considered as the significance level and independent t-test was used for comparison.

\section{Results:}

The subjects were studied in two groups of 30 each, where one group was treated with ketorolac and the other with methadone. The group treated with ketorolac had 17 men and 13 women and in methadone group 16 men and 14 women. The subjects in the two groups did not show statistically significant differences while compared with each other in terms of gender. The mean age of the subjects in the group treated with ketorolac was $52.9 \pm 9.6$ years old, the mean age of methadone-treated group was $54.6 \pm 5.3$, and the two groups did not show significant differences in terms of age compared with each other.

The mean systolic and diastolic blood pressures at baseline in the group treated with ketorolac and methadone at different time gaps are presented in Figurea,b respectively. The obtained values did not revealed any statistically signif icant difference in comparison with each other $(\mathrm{p}>0.05)$.

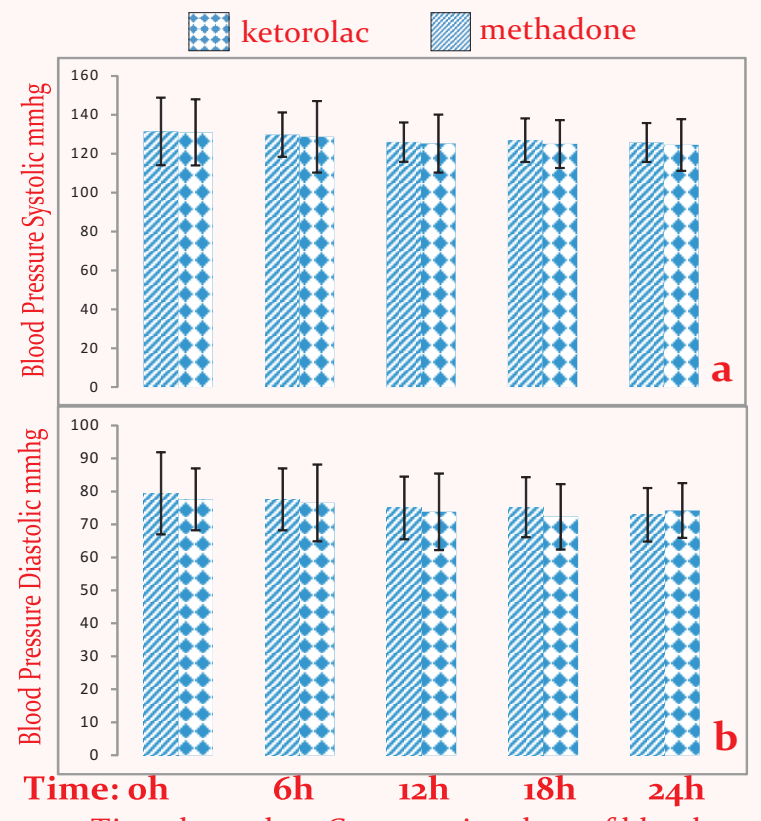

Figure-1: Time dependent Comparative chart of blood presures after treated with ketorolac and methadone a) Systolic b) Diastolic 


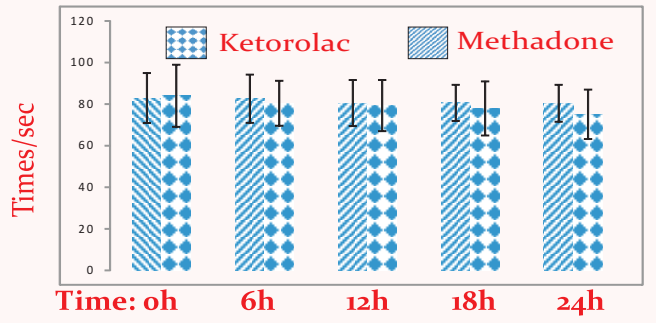

Figure-2: Time dependent Comparion of heart rate after treated with ketorolac and methadone. Statistically no significant differences.

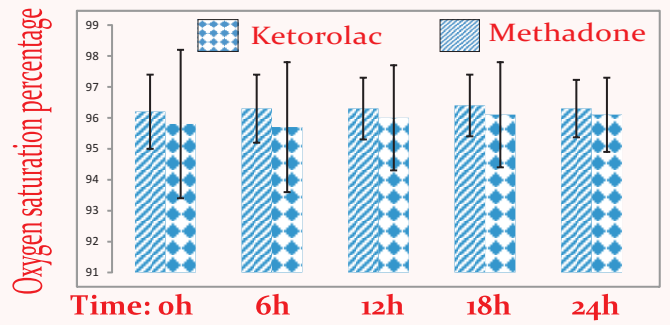

Figure-3: Time dependent Comparison of oxygen saturation percentage after treated with ketorolac and methadone Statistically no significant differences.

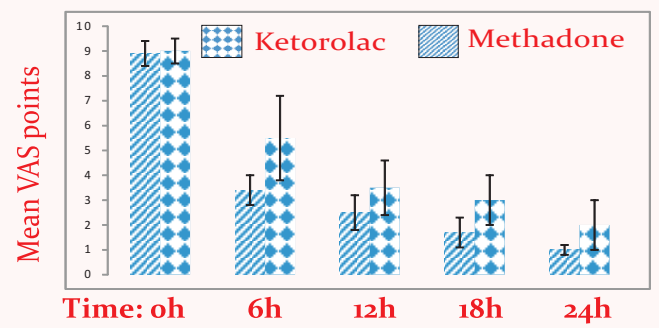

Figure-4: Time dependent Comparison of Mean VAS after treated with Ketorolac and Methadone. Statistically no significant differences.

Simialrly other studied parameters viz., heart rate, Oxygen saturation percentage, VAS scale were also not revealed any statistical diiferences between the two groups teated with Ketorolac and Methadone and which are shown in Figure 2 to 4 respectively.

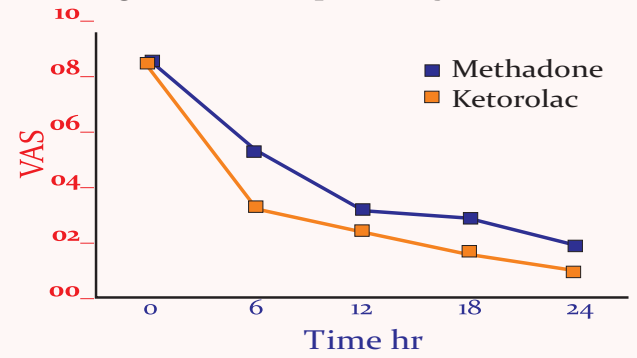

Figure-5: Comparing mean VAS for the treatment groups

At $6,12,18$, and $24 \mathrm{~h}$ later, the mean of VAS in the group treated with ketorolac was statisticaly higher than the methadone-treated group $(\mathrm{p}<0.05)($ Fig. $=5)$.

At baseline, $100 \%$ of the methadone and ketorolac treated patients did not receive Morphine sulfate, and 6 hours after surgery, $22(73.3 \%)$ subjects treated with methadone and 2 patients $(6.7 \%)$ treated with ketorolac underwent Morphine sulfate treatment. Then 12 hours after surgery 1 (3.3\%) subject treated with methadone and 1 $(3.3 \%)$ subject treated with ketorolac underwent morphine sulfate treatment. Moreover, Then 18 hours after surgery, $9(30 \%)$ subjects treated with Methadone and no one treated with Ketorolac underwent morphine sulfate treatment. Twenty-four hours after surgery, none of the subjects of the two groups underwent Morphine sulfate treatment. Comparing the two groups at baseline and 12 and 24 hours after surgery showed no statistically significant differences ( $\mathrm{p}>0.05$ ). On 6 and 18 hours after surgery, the groups treated with Ketorolac received less Morphine sulfate compared to Methadone-treated group $(\mathrm{p}<0.05)$, which was revealed statistically significant difference (Table-1).

\begin{tabular}{lllllll}
\multicolumn{6}{c}{ Table-1: Comparing morphine consumption by the groups } \\
treated with methadone and ketorolac
\end{tabular}

*Statistically not signif icant

Twenty-five $(83.3 \%)$ of the patients treated with Ketorolac showed no complications, 3 (10\%) subjects felt headache and $2(6.7 \%)$ subjects felt nausea. Moreover, $100 \%$ of those treated with methadone suffered complications: $6(20 \%)$ felt dry mouth and drowsiness, 5 (16.7\%) dry mouth alongwith palpitations, 3 (10\%) dry mouth and nausea, and $3(10 \%)$ drowsiness and headache. Moreover, 2 (6.7\%) subjects felt nausea, 2 (6.7\%) dry mouth, $2(6.7 \%)$ nausea and palpitations, 1 (3.3\%) drowsiness and nausea, $1(3.3 \%)$ nausea and headache, 1 (3.3\%) dry mouth and headache, 1 (3.3\%) dry mouth, headache and drowsiness, 1 (3.3\%) dry mouth, nausea and palpitations, $1(3.3 \%)$ nausea, headache and palpitations, 1 (3.3\%) nausea and palpitations. The complications of Methadone-treated patients were significantly higher compared to those treated with Ketorolac $(\mathrm{p}<0.05)$.

\section{Discussion:}

Pain is the most common sign making the person use emergency medical assistance and this is a fact that does not depend on age, gender and culture. The study was conducted to compare the effect of intravenous ketorolac to IM methadone regarding analgesia after spinal surgery. Currently, in America and Europe, ketorolac ampoule is widely used as a healing agent because of its high analgesic strength, so that in controlling most of the acute pains, its analgesic effect is similar to the injectable opioids (such as Morphine and Methadone). Not having respiratory depression, lack of creating dependence, and long-lasting 
analgesic effect are of the most important advantages of Ketorolac ampoules compared to injectable opioids. Elia et al. (2005) argued that NSAIDs have a better effect on pain relief compared to opioids. Shankariah et al. (2012) compared the effect of IM injection of Ketorolac and Tramadol, and found that both of these drugs reduced pain intensity from 2 to 24 hours postoperatively, but tramadol was always more effective in pain control compared to Ketorolac. Although ketorolac and methadone both reduce pain, we found in our study, Ketorolac reduced more pain as compared to methadone. Moreover, regarding VAS, the patients treated with Ketorolac received a lower score compared to those treated with Methadone, which showed statistically significant differences. Marret et al. (2005) stated that NSAIDs produced less nausea, vomiting, and drowsiness compared with opioids. The results of De Oliveira et al. (2012) reported that single-dose administration of Ketorolac increased postoperative systemic analgesia, and taking drugs such as morphine reduced complications such as nausea and vomiting. In our study, complications such as a headache, palpitations, dry mouth, nausea, and drowsiness were lower in the Ketorolac-treated group compared to the Methadone-treated group, showing a signif icant difference between the two groups. This could be argued that fewer complications of ketorolac compared with Methadone could be a factor in quicker returns to the daily routine work of the people. In a study regarding the anti-inflammatory effect of NSAIDs, on reducing the complications of morphine, Emad et al. (2011) concluded that anti-inflammatory drugs and COX inhibitors reduced morphine consumption within 24 hours, so ketorolac reduced the use of morphine for analgesia. While comparing the intravenous injection of ketorolac with the placebo group in lower back surgery and laminectomy, Cassinelli et al. (2008) reported the need for less postoperative morphine and better pain scores in the Ketorolac administration group. In our study, morphine consumption in the ketorolac-treated group was lower as compared to the Methadone-treated group with statistically significant differences between them. The vital signs of patients, including blood pressure, heart rate and oxygen saturation in the groups treated with ketorolac and methadone in different hours showed no significant differences. This was not discussed enough in other relevant studies, and thus our study could be a pioneer for further research in this stream.

\section{Conclusion:}

The results of the study identified that intravenous ketorolac as compared to IM Methadone is more effective in the management of acute postoperative pain and has fewer complications and less physical dependency. Nevertheless, Ketorolac did not decrease postoperative morphine usage compared with Methadone. Finally, as IM
Methadone is painful for the patient, we intend to use intravascular Methadone in the subsequent studies, but as here we understood that Ketorolac could be as effective as opioids in analgesia, it is better to use other groups of drugs at the first line, such as non-steroid anti-inflammatory drugs that have fewer complications.

\section{Acknowledgment:}

We would like to thank the Clinical Research Development Unit of Peymanieh Educational and Research and Therapeutic Center of Jahrom University of Medical Sciences for providing facilities.

\section{References:}

Cassinelli, E.H., Dean, C.L., Garcia, R.M., Furey, C.G. \& Bohlman, H.H. (2008): Ketorolac use for postoperative pain management following lumbar decompression surgery: a prospective, randomized double-blinded, placebocontrolled trial. Spine (Phila Pa 1976)., 33(12):1313-1317.

De Oliveira, G.S. Jr., Agarwal, D. \& Benzon, H.T. (2012): Perioperative single dose Ketorolac to prevent postoperative pain: a meta-analysis of randomized trials. Anesth. Analg. 114(2):424-433.

Domer, F. (1990): Characterization of the analgesic activity of Ketorolac in mice. Eur J Pharmacol., 177(3):127-135.

Elia, N., Lysakowski, C. \& Tramer, M.R. (2005): Does multimodal analgesia with acetaminophen, nonsteroidal antiinflammatory drugs, or selective cyclooxygenase-2 inhibitors and patient-controlled analgesia morphine offer advantages over morphine alone? Meta-analyses of randomized trials. Anesthesiology. 103(6):1296-1304.

Garrido, M.J. \& Trocóniz, I.F. (1999): Methadone: a review of its pharmacokinetic/pharmacodynamic properties. I. Pharmacol. Toxicol. Methods., 42(2):61-66.

Gillis, J.C. \& Brogden, R.N. (1997): Ketorolac. A reappraisal of its pharmacodynamic and pharmacokinetic properties and therapeutic use in pain management. Drugs, 53(1):139-188.

Halperin, D.L., Koren, G., Attias, D., Pellegrini, E., Greenberg, M.L. \& Wyss, M. (1989): Topical skin anesthesia for venous, subcutaneous drug reservoir and lumbar punctures in children. Pediatrics. 84(2):281-284.

Hyers, T.M., Tricomi, S.M. \& Liao, J.J. (1992): Inhibition of some human neutrophil functions by the cyclooxygenase inhibitor Ketorolac tromethamine. L. Leukoc. Biol., 51(5):490-495.

Marret, E., Kurdi, O., Zufferey, P. \& Bonnet, F. (2005): Effects of no steroidal anti-inflammatory drugs on patient-controlled analgesia morphine side effects: meta-analysis of randomized controlled trials. Anesthesiology, 102(6):1249-126o.

Maund, E., McDaid, C., Rice, S., Wright, K., Jenkins, B., Woolacott, N., (2011): Paracetamol and selective and non-selective nonsteroidal anti-in?ammatory drugs for the reduction in morphine-related side-effects after major surgery: a systematic review. Br. J. Anaesth.,106(3):292-297.

Miller, R.D., Eriksson, L.I., Fleisher, L.A., Wiener-Kronish, J.P., Young, W.L. (2014): Miller's Anesthesia. Pub. by: Elsevier Health Sciences. p. 3576.

Shankariah, M., Mishra, M., Kamath, R.A. (2012): Tramadol versus Ketorolac in the treatment of postoperative pain following maxillofacial surgery. L. Maxillofac. OralSurg., 11(3):264-270. 